\title{
THE EDIT DISTANCE FUNCTION OF SOME GRAPHS
}

\author{
Yumei $\mathrm{Hu}^{a}$, Yongtang $\mathrm{SHI}^{b}$ \\ AND \\ YARONG WeI ${ }^{1, a}$ \\ ${ }^{a}$ School of Mathematics \\ Tianjin University, Tianjin 300072, China \\ ${ }^{b}$ Center of Combinatorics and LPMC \\ Nankai University, Tianjin 300071, China \\ e-mail: huyumei@tju.edu.cn \\ shi@nankai.edu.cn
}

\begin{abstract}
The edit distance function of a hereditary property $\mathscr{H}$ is the asymptotically largest edit distance between a graph of density $p \in[0,1]$ and $\mathscr{H}$. Denote by $P_{n}$ and $C_{n}$ the path graph of order $n$ and the cycle graph of order $n$, respectively. Let $C_{2 n}^{*}$ be the cycle graph $C_{2 n}$ with a diagonal, and $\widetilde{C_{n}}$ be the graph with vertex set $\left\{v_{0}, v_{1}, \ldots, v_{n-1}\right\}$ and $E\left(\widetilde{C_{n}}\right)=E\left(C_{n}\right) \cup\left\{v_{0} v_{2}\right\}$. Marchant and Thomason determined the edit distance function of $C_{6}^{*}$. Peck studied the edit distance function of $C_{n}$, while Berikkyzy et al. studied the edit distance of powers of cycles. In this paper, by using the methods of Peck and Martin, we determine the edit distance function of $C_{8}^{*}, \widetilde{C_{n}}$ and $P_{n}$, respectively.
\end{abstract}

Keywords: edit distance, colored regularity graphs, hereditary property, clique spectrum.

2010 Mathematics Subject Classification: 05C35.

\section{REFERENCES}

[1] N. Alon, Testing subgraphs in large graphs, Random Structures Algorithms 21 (2002) 359-370. doi:10.1002/rsa.10056

\footnotetext{
${ }^{1}$ Corresponding author.
} 
[2] N. Alon, A. Duke, H. Lefmann, V. Rödl and R. Yuster, The algorithmic aspects of the regularity lemma, J. Algorithms 16 (1994) 80-109.

doi:10.1006/jagm.1994.1005

[3] N. Alon, M. Krivelevich, E. Fischer and M. Szegedy, Efficient testing of large graphs, in: Proc. 40th Symposium on Foundations of Computer Science) (IEEE Press, 1999) $656-666$.

[4] N. Alon and U. Stav, What is the furthest graph from a hereditary property?, Random Structures Algorithms 33 (2008) 87-104.

doi: $10.1002 /$ rsa.20209

[5] M. Axenovich, A. Kézdy and R. Martin, On the editing distance of graphs, J. Graph Theory 58 (2008) 123-138.

doi:10.1002/jgt.20296

[6] J. Balogh and R. Martin, Edit distance and its computation, Electron. J. Combin. 15 (2008) \#R20.

[7] Z. Berikkyzy, R. Martin and C. Peck, On the edit distance of powers of cycles, submitted. arXiv: 1509.07438

[8] J.A. Bondy and U.S.R. Murty, Graph Theory (New York, Springer, 2008).

[9] D. Chen, O. Eulenstein, D. Fernández-Baca and M. Sanderson, Supertrees by flipping, in: O.H. Ibarra, L. Zhang (Eds.) Computing and Combinatorics, Lectures Notes in Comput. Sci. 2387 (2002) 391-400.

doi:10.1007/3-540-45655-4_42

[10] E. Marchant and A. Thomason, Extremal graphs and multigraphs with two weighted colours, in: Fete of Combinatorics and Computer Science, Springer (2010) 239-286. doi:10.1007/978-3-642-13580-4_10

[11] R. Martin, The edit distance in graphs: Methods, results, and generalizations, in: Recent Trends in Combinatorics, Springer (2016) 31-62.

doi:10.1007/978-3-319-24298-9_2

[12] R. Martin, On the computation of edit distance functions, Discrete Math. 338 (2015) 291-305. doi:10.1016/j.disc.2014.09.005

[13] R. Martin, The edit distance function and symmetrization, Electron. J. Combin. 20 (2013) \#P26.

[14] R. Martin and T. McKay, On the edit distance from $K_{2, t}$-free graphs, J. Graph Theory 77 (2014) 117-143. doi:10.1002/jgt.21777

[15] C. Peck, On the Edit Distance from a Cycle- and Squared Cycle-Free Graph (Master's Thesis, Iowa State University, 2013).

Received 5 November 2017

Revised 11 May 2018

Accepted 11 May 2018 